\title{
Beiträge zur Kenntniss der Doppelchromate von
}

\author{
Josef Zehenter.
}

Aus dem chemischen Laboratorium der k. k. Oberrealschule in Innsbruck.

(Vorgelegt in der Sitzung am 4. Februar 1897.)

\section{Einwirkung von Natriumcarbonat auf Kaliumdichromat.}

Im 62. Bande (A. F.) des Journals für praktische Chemie findet sich S. 261 eine Mittheilung von Samuel Johnson über eine leichte Bereitungsweise des neutralen Natriumchromats. Er stellt es dar, indem eine Lösung von Kaliumdichromat mit Natriumcarbonat gesättigt und hierauf bei $0^{\circ}$ verdunstet wird. Nach seinen Angaben enthält dasselbe keine Spur Kalium und ist seine Zusammensetzung $\mathrm{Na}_{2} \mathrm{CrO}_{4}+10 \mathrm{H}_{2} \mathrm{O}$.

In der Absicht, $z u$ einem Doppelsalze von der Formel $\mathrm{K}_{2} \mathrm{CrO}_{4}+\mathrm{Na}_{2} \mathrm{CrO}_{4}$ zu gelangen, wurde der obige Versuch unter etwas geänderten Bedingungen wiederholt und dabei folgende Beobachtungen gemacht:

Eine siedend heisse Lösung von Kaliumdichromat gab nach dem Neutralisiren mit Soda, welche in einer Menge entsprechend derGleichung $\mathrm{K}_{2} \mathrm{Cr}_{2} \mathrm{O}_{7}+\mathrm{Na}_{2} \mathrm{CO}_{3}=\mathrm{K}_{2} \mathrm{CrO}_{4}+\mathrm{Na}_{2} \mathrm{CrO}_{4}$ $+\mathrm{CO}_{2}$ zur Verwendung kam, eine schwefelgelbe Flüssigkeit, die nach der zuerst über freiem Feuer und dann im Vacuum über Schwefelsäure vorgenommenen Concentration theils platten-, theils säulenförmige, oft $z u$ Büscheln vereinigte Krystalle lieferte. Dieselben zeigten, auf $150-160^{\circ}$ erhitzt, keinen Gewichtsverlust, und die bei der Analyse erhaltenen Zahlen entsprachen einem Doppelsalze von der Formel $3 \mathrm{~K}_{2} \mathrm{CrO}_{4}+\mathrm{Na}_{2} \mathrm{CrO}_{4}$. 
In 100 Theilen:

$\begin{array}{lrr}\mathrm{Cr} \ldots \ldots & \underbrace{\text { Gefunden }}_{27 \cdot 91} & \underbrace{\text { Berechnet }}_{27 \cdot 95} \\ \mathrm{~K} \ldots \ldots . & 31 \cdot 61 & 31 \cdot 50 \\ \mathrm{Na} \ldots \ldots & 6 \cdot 15 & 6 \cdot 19\end{array}$

100 Theile Wasser vermögen bei $14^{\circ}$ C. $64 \cdot 2$ Theile des Salzes zu lösen, das specifische Gewicht wurde in wasserfreiem Benzol bei $15^{\circ} \mathrm{C}$. gleich $2 \cdot 719$ gefunden.

Die erhaltenen Krystalle entsprechen ihrer Zusammensetzung nach dem Doppelsalze, das von Hauer ${ }^{1}$ durch freiwilliges Verdunsten von Kalium- und Natriumchromat erhalten wurde. Ros $\mathrm{e}^{2}$ hat es durch Zusammenschmelzen von Kaliumdichromat und Soda dargestellt, und Wy roub off ${ }^{3}$ hat dasselbe nach Hauer nochmals krystallographisch untersucht.

Bei weiterer Concentration der Mutterlauge scheiden sich zu Drusen vereinigte, plattenförmige, zart gebaute Krystalle ab, welche sich bei der Untersuchung gleich zusammengesetzt dem früheren Doppelsalze erwiesen, nur dass sie noch ein halbes Molekül Krystallwasser enthielten.

Bei $150^{\circ}$ getrocknet verloren die vollkommen lufttrockenen, von verschiedenen Darstellungen herrührenden Krystalle 1.5, 1.44 und $1.1 \%$ Wasser, während die Formel $3 \mathrm{~K}_{2} \mathrm{CrO}_{4}+\mathrm{Na}_{2} \mathrm{CrO}_{4}$ $+1 / 2 \mathrm{H}_{2} \mathrm{O}$ für $1 / 2 \mathrm{H}_{2} \mathrm{O} 1 \cdot 19 \%$ verlangt. Eine Chromsäurebestimmung, in der lufttrockenen Substanz ausgeführt, ergab $53.42 \%$, während der Theorie 53.08\% entsprechen. Die Analyse des wasserfreien Körpers gab Zahlen, die mit der Formel $3 \mathrm{~K}_{2} \mathrm{CrO}_{4}$ $+\mathrm{Na}_{2} \mathrm{CrO}_{4}$ übereinstimmten.

In 100 Theilen:

\begin{tabular}{|c|c|c|}
\hline & Gefunden & Berechnet \\
\hline & $28 \cdot 02$ & $27 \cdot 95$ \\
\hline K & $31 \cdot 22$ & $31 \cdot 50$ \\
\hline $\mathrm{Na}$ & $6 \cdot 77$ & $6 \cdot 19$ \\
\hline
\end{tabular}

1 Journal für prakt. Chemie, A. F., 83. Bd., S. 356.

2 Poggendorff's Anna1, 52. Bd., S. 585.

3 Zeitschrift für Krystallographie, 8. Bd., S. 635. 
100 Theile Wasser lösen bei $14^{\circ}$ C. $66 \cdot 4$ Theile des Salzes; das specifische Gewicht, bestimmt durch Wägen in Benzol, war bei $15^{\circ} \mathrm{C}$. gleich $2 \cdot 575$.

Die Mutterlauge dieses wasserhältigen Doppelsalzes lieferte nach längerem Stehen neben geringen Mengen der früheren Körper eisblumenartig aussehende Krystalle, welche sich oft plötzlich beim Schütteln der syrupösen Mutterlauge bildeten und mit Salzsäure ganz geringes Aufbrausen zeigten. $\mathrm{Zu}$ ihrer Reinigung umkrystallisirt, stellten sie dann schwefelgelbe, oft stark verfilzte Nadeln dar, welche sehr leicht löslich sind. Die Analyse gab Zahlen, die für $\mathrm{Na}_{2} \mathrm{CrO}_{4}+4 \mathrm{H}_{2} \mathrm{O}$ stimmen.

Die lufttrockene Substanz verliert bereits beim längeren Liegen an der Luft, noch mehr im Vacuum über Schwefelsäure, vollständig beim Trocknen auf $150^{\circ} \mathrm{ihr}$ Krystallwasser.

In 100 Theilen:

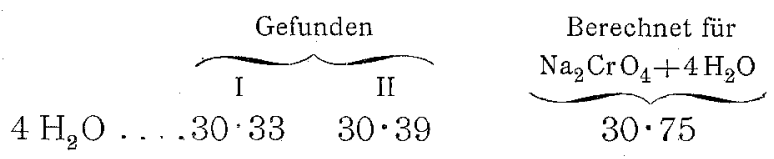

Die Analyse der wasserfreien Substanz ergab:

\begin{tabular}{|c|c|c|}
\hline & Gefunden & Berechnet \\
\hline & $31 \cdot 99$ & $32 \cdot 11$ \\
\hline & $27 \cdot 95$ & $28 \cdot 44$ \\
\hline
\end{tabular}

Wie H. Traube ${ }^{1}$ mittheilt, konnte er ebenfalls die Existenz eines Natriumchromates mit 4 Mol. Krystallwasser, welche zuerst von Gernez beobachtet, von Retgers aber bezweifelt wurde, bestätigen. $\mathrm{Zu}$ einem ähnlichen Resultate ist auch Wyroub off ${ }^{2}$ gelangt.

Die Ausbeute der hier erwähnten Salze betrug unter Zugrundelegung der Menge des angewandten Kaliumdichromates ungefähr $40 \%$ an dem erstbesprochenen Salze, $30 \%$ an dem in zweiter Stelle genannten und $20 \%$ an dem letzterwähnten, wobei zu bemerken ist, dass die letzten Mutterlaugen nicht mehr Berücksichtigung fanden.

1. Zeitschrift für Krystallographie, 22. Bd., S. 138.

2 Desgl. 8. Bd., S. 626. 
Der Versuch der Neutralisation von Natriumdichromat mit Kaliumcarbonat lieferte dieselben Producte.

Vergleicht man die durchgeführten Versuche mit dem von Johnson angestellten, so sieht man, dass ein Natriumchromat mit 10 Mol. Wasser sich bei den ersteren nicht bildet, sondern dass ausser zwei Doppelchromaten noch Natriumchromat mit 4 Mol. Wasser erzeugt wird. Es scheirit also die Temperatur, bei der die Sättigung und das Verdunsten vorgenommen wird, von wesentlichem Einfluss auf die sich bildenden Producte $z u$ sein.

\section{Einwirkung von Kaliumcarbonat auf Ammoniumdichromat.}

An der früher erwähnten Stelle machte Johnson auch eine Mittheilung über chromsaures Kaliammoniak, welches er durch Versetzen einer warm gesättigten Lösung von Kaliumdichromat mit concentrirtem Ammoniak und Verdunsten bei niedriger Temperatur erhielt. Das Doppelsalz hat nach ihm die Formel $\mathrm{K}_{2} \mathrm{CrO}_{4}+\left(\mathrm{NH}_{4}\right)_{2} \mathrm{CrO}_{4}$, gibt an der Luft Ammoniak ab und wird gelbroth. Nach Etapd ${ }^{1}$ wird dieses Salz durch Vermischen heisser Lösungen von Ammoniumchlorid und Kaliumchromat und nachherigem Verdunsten in langen, gelben und glänzenden Nadeln gebildet, welche 1 Mol. Krystallwasser enthalten.

Es wurde nun untersucht, wie sich Ammoniumdichromat gegenüber Kaliumcarbonat verhält. Schwierigkeiten bereitete dabei die Eigenschaft des erhaltenen Körpers, leicht Ammoniak abzuspalten, so dass ein Umkrystallisiren, ein Abdampfen auf dem Wasserbade oder im Vacuum, ja selbst blosses Stehenlassen der wässerigen Lösung vermieden werden 'muss. Nach einer Reihe von Versuchen wurde folgendes Verfahren eingeschlagen:

Eine concentrirte wässerige Lösung von Ammoniumdichromat wurde bei gewöhnlicher Temperatur mit so viel Kaliumcarbonat versetzt, als der Gleichung $\left(\mathrm{NH}_{4}\right)_{2} \mathrm{Cr}_{2} \mathrm{O}_{7}+$ $\mathrm{K}_{2} \mathrm{CO}_{3}=2 \mathrm{KNH}_{4} \mathrm{CrO}_{4}+\mathrm{CO}_{2}$ entspricht, die schwach nach Ammoniak riechende Flüssigkeit filtrirt und hierauf mit der

1 Compt. rend., 85. Bd., p. 443. 
fünffachen Menge Alkohol von 90-95\% versetzt; der erhaltene Krystallbrei filtrirt, mit Alkohol gewaschen, gut abgesaugt, abgepresst und getrocknet. Zur Reinigung wurde die Masse nochmals in möglichst wenig Wasser gelöst und die Fällung mit Alkohol wiederholt. Der nun erhaltene schwefelgelbe Krystallbrei bestand, unter dem Mikroskop betrachtet, aus feinen, oft verfilzten, einheitlich aussehenden Nädelchen, die in vollkommen trockenem Zustande ziemlich beständig sind, im feuchten aber unter Abspaltung von Ammoniak bald ihre Form verlieren.

Die Analyse dieser Krystalle führte zu einem Doppelsalze von der Formel $3 \mathrm{~K}_{2} \mathrm{CrO}_{4}+2\left(\mathrm{NH}_{4}\right)_{2} \mathrm{CrO}_{4}$.

In 100 Theilen:

\begin{tabular}{|c|c|c|}
\hline \multicolumn{3}{|c|}{ Gefunden } \\
\hline$I$ & II & Berechnet \\
\hline $\mathrm{NH}_{4} \ldots \ldots 8 \cdot 18$ & $7 \cdot 96$ & $8 \cdot 14$ \\
\hline$\ldots .26 \cdot 38$ & $25 \cdot 91$ & $26 \cdot 46$ \\
\hline$\ldots .29 \cdot 56$ & $29 \cdot 39$ & $29 \cdot 34$ \\
\hline $\mathrm{CrO}_{3} \ldots .56 \cdot 79$ & $56 \cdot 66$ & $56 \cdot 39$ \\
\hline
\end{tabular}

Die Krystalle sind in Wasser sehr leicht löslich, ihr specifisches Gewicht, in Benzol gemessen, betrug 2.403 bei $15^{\circ} \mathrm{C}$. Kocht man sie mit der nöthigen Menge Wasser, so verschwindet die schwefelgelbe Färbung der Lösung, um unter reichlicher Abspaltung von Ammoniak einer rothen Platz zu machen. Aus der rückständigen Flüssigkeit krystallisirt Kaliumdichromat, aus der Mutterlauge desselben Kaliumchromat aus. Bei einem quantitativ. durchgeführten Versuche lieferten $0.927 \mathrm{~g}$ des Doppelsalzes nach dem Verdampfen des Wassers und Trocknen des Rückstandes $0.808 \mathrm{~g}$, während die Theorie nach der Formelgleichung $2\left(\mathrm{NH}_{4}\right)_{2} \mathrm{CrO}_{4}+3 \mathrm{~K}_{2} \mathrm{CrO}_{4}=2 \mathrm{~K}_{2} \mathrm{Cr}_{2} \mathrm{O}_{7}+\mathrm{K}_{2} \mathrm{CrO}_{4}+$ $4 \mathrm{NH}_{3}+2 \mathrm{H}_{2} \mathrm{O} 0.818 g$ verlangt. Beim Erhitzen der Substanz für sich allein oder im Wasserstoffstrome auf $160^{\circ}$ findet gleichfalls Entweichen von Ammoniak und Wasser in einer Menge statt, wie sie obiger Formelgleichung entspricht. $11.72 \%$ Verlust ergab der Versuch, $11.73 \%$ verlangt die Theorie. Was die Ausbeute des beschriebenen Doppelsalzes betrifft, so lässt sich 
dafür keine bestimmte Zahl angeben, da ein Theil immer durch Zersetzung im früher angedeuteten Sinne, die auch bei gewöhnlicher Temperatur eintritt, verloren geht.

\section{Einwirkung von Natriumcarbonat auf Ammonium- dichromat.}

Wyrouboff ${ }^{1}$ stellte durch Mischung der Lösungen von Natriumchromat und Ammoniumchromat und nachheriges Auskrystallisiren ein Doppelsalz von der Formel $\mathrm{NaNH}_{4} \mathrm{CrO}_{4}+$ $2 \mathrm{H}_{2} \mathrm{O}$ dar und beschrieb es in krystallographischer Hinsicht.

Es stellte sich nun heraus, dass sich dasselbe auch beim Absättigen von Ammoniumdichromat mit Natriumcarbonat bildet. Dabei wurde in ähnlicher Weise wie bei der Darstellung des vorigen Doppelsalzes verfahren. Nach dem Zusatze von Alkohol setzte sich zunächst eine ölartige Flüssigkeit ab, die aber bald zu mehr oder weniger deutlichen Krystallen erstarrte. Die Reinigung wurde gleichfalls durch Lösen in wenig Wasser und Fällen mit Alkohol erreicht. Will man möglichst grosse Krystalle erzielen, so versetzt man die wässerige Lösung nur mit so viel Alkohol, dass sich eine deutliche Trübung bildet, und lässt dann längere Zeit bei niedriger Temperatur und im Dunkeln stehen. Am Lichte tritt eine weitergehende Zersetzung ein, ähnlich jener, welche alkoholische Lösungen von Natriumund Ammoniumdichromat erleiden.

Die Analyse der lufttrockenen Substanz lieferte Zahilen, welche der Formel $\mathrm{NaNH}_{4} \mathrm{CrO}_{4}+2 \mathrm{H}_{2} \mathrm{O}$ entsprechen.

In 100 Theilen:

$\begin{array}{lrr}\mathrm{NH}_{4} \ldots \ldots & \underbrace{\text { Gefunden }}_{9 \cdot 32} & \text { Berechnet } \\ \mathrm{Na} \ldots \ldots & 12 \cdot 33 & 11 \cdot 94 \\ \mathrm{Cr} \ldots \ldots & 27 \cdot 00 & 26 \cdot 96 \\ \mathrm{CrO}_{3} \ldots \ldots & 51.63 & 51 \cdot 80\end{array}$

Beim Versuche, das Krystallwasser durch Trocknen im Vacuum über Schwefelsäure zu entfernen, wurden meist etwas

1. Zeitschrift für Krystallographie, 4. Bd., S. 417. 
$\mathrm{zu}$ hohe Zahlen erhalten, was auf eine beginnende Zersetzung schliessen lässt. So wurden in drei Versuchen 19.75, $19 \cdot 12$ und $18.80 \%$ Verlust erhalten, während die Rechnung für 2 Mol. Krystallwasser $18 \cdot 64 \%$ verlangt.

Die Krystalle sind prismenförmig, in Wasser leicht löslich, ihr specifisches Gewicht, bestimmt bei $15^{\circ} \mathrm{C}$. durch Wägen in wasserfreiem Benzol, wurde gleich 1.842 gefunden. Beim Liegen der trockenen Substanz an der Luft tritt keine Gewichtsabnahme ein; erhitzt man sie auf $150-180^{\circ}$, so findet Zersetzung statt, Ammonials und Wasser werden abgespalten, und zwar entspricht der Vorgang, wie ein quantitativ ausgeführter Versuch lehrte, wahrscheinlich der Formelgleichung $2 \mathrm{NaNH}_{4} \mathrm{CrO}_{4}=\mathrm{Na}_{2} \mathrm{Cr}_{2} \mathrm{O}_{7}+2 \mathrm{NH}_{3}+\mathrm{H}_{2} \mathrm{O}$; der Gewichtsverlust betrug einschliesslich des Krystallwassers $32 \cdot 61 \%$, während sich $32.04 \%$ berechnen. Auch beim Kochen der wässerigen Lösung geht eine ähnliche Umwandlung vor sich.

\section{Einwirkung von Lithiumcarbonat auf Kaliumdichromat.}

Versuche, durch Sättigen von Ammoniumdichromat mit Lithiumcarbonat ein den früheren Körpern analoges Doppelsalz darzustellen, führten bis jetzt zu keinem günstigen Resultate, wohl aber war dies der Fall, als an Stelle von Ammoniumdichromat Kaliumdichromat zur Verwendung kam. Dabei wurde wie bei der Gewinnung des unter I erwähnten Doppelsalzes vorgegangen, die gesättigte Lösung einfach im Vacuum über Schwefelsäure verdunstet. Zunächst krystallisirte neben geringen Mengen von Kaliumdichromat Kaliumchromat aus, und erst dann wurden schön goldgelbe, bald grössere, bald kleinere nadelförmige Krystalle erhalten, die aber schwer luftrocken zu bekommen sind, da sie bald zerfliessen. Die so gut als möglich durch Abpressen lufttrocken gemachte Substanz verlor beim Stehen über Schwefelsäure ohne Anwendung des Vacuums einmal $2 \cdot 11 \%$, ein $z$ weites Mal $2.99 \%$, während die Formel $\mathrm{K}_{2} \mathrm{CrO}_{4}+\mathrm{Li}_{2} \mathrm{CrO}_{4}+1 / 2 \mathrm{H}_{2} \mathrm{O}$ für $1 / 2 \mathrm{H}_{2} \mathrm{O} 2 \cdot 7 \%$ verlangt. Beim Trocknen im Vacuum und bei höherer Temperatur findet keine Gewichtsabnahme mehr statt. Die über Schwefelsäure getrocknete Substanz gab bei der Analyse Zahlen, die der Formel $\mathrm{K}_{2} \mathrm{CrO}_{4}+\mathrm{Li}_{2} \mathrm{CrO}_{4}$ entsprechen. 


$\begin{array}{llr}\text { I } & \text { Gefunden } & \underbrace{\text { Berechnet }}_{\text {II }} \\ \mathrm{K} \ldots \ldots .24 \cdot 12 & - & 4 \cdot 13 \\ \mathrm{Li} \ldots \ldots .4 \cdot 38 & - & 4 \cdot 34 \\ \mathrm{C} . \ldots \ldots .32 \cdot 01 & 32 \cdot 25 & 32 \cdot 10\end{array}$

Das specifische Gewicht, durch Wägen in Benzol bei $15^{\circ}$ C. bestimmt, war gleich $2 \cdot 539$.

In der Literatur findet sich eine Abhandlung von H.Tra ube, ${ }^{1}$ in der er die Analyse und Krystallform einiger Lithiumsalze mittheilt. Unter Anderem beschreibt er Mischkrystalle von der Zusammensetzung $\left[9 \mathrm{KLiSO}_{4}+\mathrm{KLiCrO}_{4}\right]$; ein Kaliumlithiumchromat allein konnte er in deutlichen Krystallen nicht erhalten. Erwähnt mag noch werden, dass Traube ${ }^{2}$ durch Abstumpfen einer Lösung von Natriumdichromat mit Lithiumcarbonat Krystalle von der Formel $3\left(\mathrm{Na}_{2} \mathrm{CrO}_{4}+3 \mathrm{H}_{2} \mathrm{O}\right)+\mathrm{Li}_{2} \mathrm{CrO}_{4}+3 \mathrm{H}_{2} \mathrm{O}$ erhielt und dieselben auch gemessen hat.

Zum Schlusse dieser Mittheilungen sei noch erwähnt, dass als Grundlage zur Berechnung der einzelnen Zahlen die Atomgewichte nach Lothar Meyer-Seubert in Anwendung kamen, nur für das Chrom fand die neueste von Meineke ${ }^{3}$ bestimmte Zahl 51.94 Benützung. Die Chrombestimmung wurde auf gewöhnliche Weise nach vorhergegangener Reduction durch Fällen mit Ammoniak ausgeführt; das erhaltene Chromhydroxyd jedoch, da es stets etwas Alkali zurïckhält, ${ }^{4}$ nochmals in Salzsäure gelöst und neuerdings gefällt, worauf es sich für vorliegenden $Z$ weck als genügend rein erwies. Die Bestimmung der Chromsäure geschah auf massanalytischem Wege nach der Methode von Schwarz ${ }^{5}$ und wurde bei den Ammoniumdoppelsalzen mit der des Ammoniaks vereinigt.

1 Zeitschrift für Krystallographie, 24. Bd., S. 168.

2 Neues Jahrbuch für Mineralogie, 1894, I. Bd., S. 189.

3 Lieb. Ann., 261. Bd., S. 367.

4 Berl. Ber., 1889, S. 2038.

Fresenius, Quant. Analyse, 6. Aufl., I. Bd., S. 381. 